\title{
Mitarbeitergespräche führen und gestalten
}

\author{
Eva Douma
}

Gespräche zwischen Mitarbeitern und Vorgesetzten sind Voraussetzung und Grundlage einer erfolgreichen und zufrieden stellenden Zusammenarbeit in Organisationen. Das Gespräch als wechselseitiger Austausch kann Missverständnisse und Konflikte in der Zusammenarbeit klären. Und nicht zuletzt soll das Mitarbeitergespräch die Arbeitszufriedenheit und damit den Unternehmenserfolg fördern.

Auch wenn in sozialen Organisationen viel miteinander geredet wird, so ist das strukturierte Mitarbeitergespräch ein immer noch vernachlässigtes Führungsinstrument. Zufriedene Mitarbeiterinnen und Mitarbeiter sind jedoch die Basis guter Sozialer Arbeit. Nur wenn sich die Mitarbeitenden selbst ernst genommen fühlen, dann sind sie auch auf Dauer bereit, die Klienten und Kunden adäquat $\mathrm{zu}$ versorgen.

Immer flachere Hierarchien in den Einrichtungen führen zudem dazu, dass die einzelnen Mitarbeiterinnen und Mitarbeiter selbstverantwortlicher Handeln und entscheiden müssen. Nicht nur um ein einheitliches Handeln des Betriebes sicher zu stellen, sind klare Absprachen über das angestrebte Ergebnis zu treffen.

Was die notwendigen Voraussetzungen für die erfolgreiche Durchführung von Mitarbeitergesprächen sind, wird im Folgenden skizziert.

\section{Erwartungen der Einrichtung}

Aus der Sicht der Einrichtung gibt es verschiedene Anlässe für ein Gespräch zwischen Mitarbeiterenden und Vorgesetzten. Mitarbeitergespräche thematisieren Probleme in der Arbeit des Mitarbeiters (Kritikgespräch) oder zollen ihm Lob (Anerkennungsgespräch). Es soll eine allgemeine Personalbeurteilung erstellt werden (Beurteilungsgespräch) oder die Weiterbildungserfordernisse der Mitarbeiterinnen und Mitarbeiter werden festgehalten (Entwicklungsgespräch).

Grundsätzlich ist zwischen geplanten, regelmäßig wiederkehrenden (Jahres-) Gesprächen und ad hoc geführten Gesprächen zu einem bestimmten Anlass (z. B. Abmahnung, Kündigung, Versetzung, Rückkehrgespräch nach einer längeren Krankheit) zu unterscheiden.

Unabhängig von Form und Anlass des Mitarbeitergespräches geht es für die Einrichtung darum, vor allem einen reibungslosen Betriebsablauf sicherzustellen. Für den Betrieb steht letztendlich immer die Umsetzung der Unternehmensziele im Mittelpunkt der Gespräche.

\section{Erwartungen der Mitarbeiter}

Aus der Sicht der Mitarbeiterinnen und Mitarbeiter werden Personalgespräche, insbesondere wenn es sich dabei um Beurteilungsgespräche, Zielvereinbarungsgespräche und dergleichen handelt, oft mit einer gewissen Skepsis betrachtet. »Was will der Vorgesetzte von mir? « Ein gut geführtes Mitarbeitergespräch stellt jedoch für die Mitarbeiterinnen und Mitarbeiter eine Chance dar, ihren Standpunkt im Unternehmen zu beleuchten. Sie können eine wertvolle Hilfe für die Planung ihres weiteren Berufsweges im Betrieb sein. Wichtig hierfür ist, dass die Mitarbeiterinnen und Mitarbeiter diese Chance erkennen und auch bekommen.

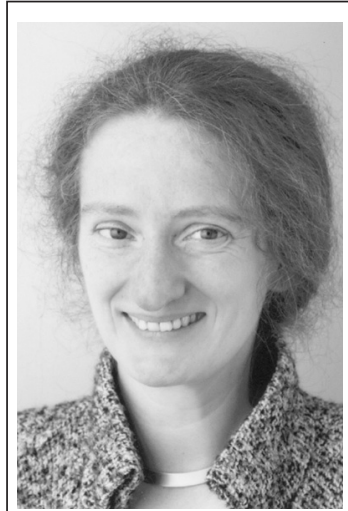

Dr. Eva Douma ist Inhaberin des Eva Douma Verlages in Berlin und Frankfurt am Main. Sie ist zudem als Trainerin und Moderatorin mit den Schwerpunkten Personal- und Organisationsentwicklung tätig. Der Beitrag ist ein überarbeiteter Auszug aus ihrem neuen Buch, das im Oktober 2006 erscheinen wird (Eva Douma: Pflegekräfte pflegen. Personalentwicklung in der ambulanten Pflege. Berlin 2006. 39,90 Euro. ISBN 3-98108361-x).

Internet http://www.douma.de

Die Mitarbeiterinnen und Mitarbeiter wollen wissen, welche Perspektiven für sie in der Einrichtung bestehen. Wie steht es um den Betrieb, welche Anforderungen werden zukünftig an mich gestellt, welche Möglichkeiten gibt es für mich?

\section{Formen der Mitarbeitergespräche}

Aus der Vielzahl von Themen und Anlässen für Gespräche in der beruflichen Praxis werden hier einige wesentliche Formen und deren Ziele zunächst im Überblick vorgestellt.

- Das Anerkennungsgespräch: Ziel eines Anerkennungsgespräches ist es, die Mitarbeiterin oder den Mitarbeiter zu loben und so zu weiteren herausragenden Leistungen anzuspornen. Anerkennung ist eine Form der immateriellen Belohnung für erbrachte überdurchschnittliche Leistungen. Lob steigert das Selbstwertgefühl der Mitarbeitenden und gibt ihnen die notwendige Sicherheit bei zukünftigen Handlungen und Entscheidungen.

- Das Kritikgespräch: Wenn Mitarbeiterinnen und Mitarbeiter 
schlechte Leistungen erbringen oder Fehler machen, kann ein konstruktives Problemgespräch eine Verhaltensänderung herbeiführen.

- Das Beurteilungsgespräch: Beim Beurteilungsgespräch handelt es sich um ein Gespräch zwischen Führungskraft und Mitarbeiter zur Personalbeurteilung im klassischen Sinn. Dies erfolgt meist dann, wenn es um Gehaltserhöhungen, Entlassungszeugnisse oder die Versetzungsmöglichkeiten von Mitarbeiterinnen und Mitarbeiter geht.

- Das Entwicklungsgespräch: Beim Entwicklungsgespräch werden die Ressourcen und Entwicklungspotenziale der Mitarbeiterinnen und Mitarbeiter beleuchtet und ihr Weiterbildungsbedarf wird abgefragt. Zukünftige Qualifizierungsnotwendigkeiten werden angesprochen. Entwicklungsgespräche dienen eher der internen Versetzung als der Weiterbildung im Job. Weiterbildung im Job lässt sich insbesondere in einem Zielvereinbarungsgespräch thematisieren und vereinbaren.

- Die Vorgesetztenbeurteilung: Die Vorgesetztenbeurteilung dient dazu, die Führungsergebnisse zu verbessern. Hierzu beurteilen die Mitarbeiterinnen und Mitarbeiter das Verhalten und die Führungsqualität der Vorgesetzten.

- Das Zielvereinbarungsgespräch: In Zielvereinbarungsgesprächen wird festgelegt, welche Ziele und Aufgaben in der nächsten Zeit anstehen und wie diese umgesetzt werden können. Angeregt werden soll ein betrieblicher Dialog und die Ermittlung von Weiterbildungszielen.

- Das Mitarbeiterjahresgespräch: Häufiger werden in Einrichtungen so genannte Mitarbeiterjahresgespräche geführt. Diese sind in der Regel eine Mischung der oben dargestellten Gesprächstypen.

\section{Inhalt und Ablauf}

Unabhängig davon, welche Art von Mitarbeitergespräch im Einzelfall geführt wird: Für alle Formen des Gespräches gelten einige Grundregeln.
Aufgabe eines jeden Mitarbeitergespräches ist es, das Verhältnis zwischen den Gesprächspartnern zu verbessern und die Qualität der Arbeit zu fördern. Erreicht werden kann dies am besten durch Offenheit, Vertrauen und gegenseitige Akzeptanz. Offenheit und Akzeptanz sind dabei keine Werte, die gesetzt werden. Sie müssen mit der Zeit entwickelt werden. Ein gut strukturiertes Verfahren kann dies fördern. Ein Mitarbeitergespräch sollte immer gut vorbereitet sein und ungestört verlaufen.

Vorgesetzte sollten berücksichtigen, dass Mitarbeitergespräche auch eine Form der Machtausübung darstellen. In der Regel sind es die Vorgesetzten, die Zeitpunkt, Inhalt, Länge und Form des Gespräches festlegen. Sie dominieren damit das Gespräch. Dies lässt sich durch die Beachtung einiger Verfahrensregeln abmildern.

Fairness ist dabei das oberste Gebot. Dies gilt insbesondere für Konfliktsituationen. Selbst dringende und kurzfristig zu führende Gespräche sollten immer angekündigt werden. Nicht nur die Vorgesetzten auch die Mitarbeiterinnen und Mitarbeiter sollten die Chance haben, sich auf das Gespräch vorbereiten zu können. Impulsive Überfälle »Frau ... kommen Sie mal in mein Büro « sind zumindest mittelfristig nicht hilfreich.

Selbstverständlich sollte für ein Mitarbeitergespräch genügend Zeit zur Verfügung stehen. Störungen sind zu vermeiden, damit das Gespräch ungestört verlaufen kann - dementsprechend sollte kein Telefon klingeln und auch niemand ins Büro platzen.

Jedes Mitarbeitergespräch verläuft in Phasen, die Sie möglichst einhalten sollten (vgl. Kießling-Sonntag, Jochem, Handbuch Mitarbeitergespräche, Berlin 2000, S. 45 ff.).

\section{Phase 1: Die Vorbereitung}

Jedes Gespräch sollte gut vorbereitet sein. Dies ist nicht nur eine Frage der Achtung gegenüber den Mitarbeiterinnen und Mitarbeitern. Ein Mitarbeitergespräch kostet viel Zeit.

Schlecht vorbereitete Gespräche sind verschwendete Zeit.

Informieren Sie Ihre Mitarbeiter darüber, dass ein Gespräch ansteht.
Legen Sie einen Gesprächstermin fest. Legen Sie fest, wie lange das Gespräch dauern soll. Seien Sie bei der Zeitplanung nicht zu eng. Eine Stunde ist schnell vorbei. Aber ein Gespräch sollte auch nicht open end geführt werden. 45 Minuten bis eine Stunde sind ein durchaus realistisches Zeitbudget.

Wenn Sie ein Mitarbeitergespräch nicht zum ersten Mal führen: Schauen Sie noch mal in Ihre Aufzeichnungen des letzten Gespräches. Was vereinbarten Sie seinerzeit? Was wurde daraus?

Legen Sie fest, was Ihr Ziel für dieses Gespräch ist. Machen Sie dies am besten schriftlich. Eine Stichpunktliste genügt durchaus. Was wollen Sie ansprechen? Was ist Ihr konkretes Ziel, welche Vereinbarung für die $\mathrm{Zu}$ kunft streben Sie an?

Wer soll das Gespräch führen? In der Regel führt der unmittelbare Vorgesetzte das Mitarbeitergespräch. In Ausnahmefällen kann es auch besser sein, wenn die übergeordnete Führungskraft das Gespräch führt. So kann es sinnvoll sein, dass bei einem Streit zwischen Fachbereichsleitung und Sozialarbeiterin das Gespräch von der Geschäftsführung geführt wird.

Jedes Mitarbeitergespräch ist in der Regel ein Vieraugengespräch. Nur in Ausnahmefällen sollten mehr als zwei Personen beteiligt sein. Wenn Sie als Vorgesetzte sich weitere Kollegen als Verstärkung holen, so sollten sie dies auch den Mitarbeiterinnen und Mitarbeitern zubilligen. Gerade bei Konfliktgesprächen kann es sinnvoll sein, das der Mitarbeiter zum Beispiel durch ein Mitglied des Betriebsrates begleitet wird.

\section{Phase 2: Das Gespräch}

Es ist sinnvoll, das Mitarbeitergespräch in verschiedenen Schritten ablaufen zu lassen.

- Kontaktphase: Eine kurze Aufwärmphase dient dazu, eine gute Gesprächsatmosphäre zu schaffen. Fallen Sie nicht mit der Tür ins Haus. Stellen sie ein positives Gesprächsklima her. Ist der Gesprächsanlass jedoch ein eher unangenehmer, dann vermeiden Sie 
Smalltalk. Schließlich ist der Anlass des Gespräches ernst. Keinesfalls sollte der Smalltalk zu lange dauern. Zwei bis drei Sätze genügen, sonst wird der Smalltalk zum Gesprächsinhalt. Das ist nicht das Ziel. Lassen Sie nicht den Schreibtisch zwischen sich und setzen Sie sich nicht auf einen höheren Stuhl als Ihre Mitarbeiter. Sehen Sie Ihr Gegenüber an, doch durchbohren Sie Ihre Gesprächspartner nicht mit Blicken.

- Informationsphase: Nennen Sie den Gesprächsanlass. Machen Sie deutlich, was das Ziel des Gespräches ist und welche Bedeutung das Gespräch für den Mitarbeiter und seine Arbeit hat. Legen Sie fest, wie viel Gesprächszeit zur Verfügung steht. Klären Sie gemeinsam mit dem Mitarbeiter, welche Themen anstehen - auch wenn Sie im Vorfeld schon darüber informierten, vielleicht ist Ihnen bei der Vorbereitung noch etwas eingefallen. Unter Umständen will auch der Mitarbeiter noch ein Thema besprechen. Erstellen Sie gemeinsam eine kleine Tagesordnung und legen Sie ein Zeitbudget für die einzelnen Punkte fest. Wenn Sie merken, dass die geplante Zeit nicht reicht, so legen Sie gemeinsam fest, welche Punkte besonders wichtig sind und in jedem Fall besprochen werden sollten, Legen Sie fest, wann und in welcher Form sie die weiteren Themen besprechen. Unter Umständen vereinbaren Sie einen weiteren Termin.

- Argumentationsphase: Trennen Sie die Argumentationsphase deutlich von der Informationsphase. Informationen sind Fakten. Argumentationen zeichnen sich durch Werturteile und Einschätzungen einer Situation aus. Klären Sie erst die Fakten, bevor Sie sie bewerten. Sagen Sie klar und deutlich, was Sie wollen, womit Sie zufrieden und womit Sie nicht zufrieden sind. Machen Sie deutlich, was Ihre zukünftigen Anforderungen an den Mitarbeiter sind. Reden Sie nicht um den heißen Brei herum. Geben Sie dem Mitarbeiter Gelegenheit Rückfragen zu stellen. Geben Sie ihm Zeit zur Stellungnahme. Wie stellt sich die Situation aus seiner Sicht dar? Was strebt er an? Klären sie gemeinsam, inwieweit es zur Umset- zung der angestrebten Ziele Ihrer Unterstützung oder der Dritter (Kollegen) bedarf. Signalisieren Sie Ihre Unterstützungsbereitschaft und unterbreiten Sie ein konkretes Unterstützungsangebot.

- Entscheidungsphase: Treffen Sie mit der Mitarbeiterin oder dem Mitarbeiter konkrete Vereinbarungen. Vereinbaren Sie Termine und klären Sie die Verantwortlichkeiten. Vereinbaren Sie insbesondere Zwischenschritte, in denen Sie sich erneut mit dem Mitarbeiter austauschen, inwieweit sich die erarbeitete Lösung oder Zielvorgabe umsetzen lässt. Wenn Sie mehrere Ziele vereinbaren, legen Sie Prioritäten fest.

- Abschlussphase: Jedes Gespräch sollte positiv beendet werden. Legen Sie gemeinsam einen Termin fest, an dem Sie die getroffenen Vereinbarungen überprüfen, vereinbaren Sie gegebenenfalls Zwischenschritte. Überprüfen Sie Ihre Vereinbarung auf ihre Machbarkeit. Eine unrealistische Vereinbarung schafft auf Dauer nur Frustration, das nächste Kritikgespräch ist vorprogrammiert. Bedanken Sie sich für die Offenheit Ihres Mitarbeiters, über sich selbst und die Hintergründe des Verhaltens gesprochen zu haben. Bedanken Sie sich auch für ein Gespräch, wenn es nicht in Ihrem Sinne verlief. Dabei sollten Sie nicht heucheln. Ein Dank für das »gute« Gespräch, wenn es ein schlechtes war, ist kontraproduktiv. Aber in jeden Fall haben Sie unterschiedliche Positionen ausgetauscht. Und selbst, wenn der Mitarbeiter nicht viel gesagt hat, so hatten Sie zumindest die Gelegenheit, Ihren Standpunkt deutlich machen. Bedanken Sie sich dafür. Denn jedes Gespräch ist die Ausgangslage für ein nächstes und dieses sollte immer noch möglich sein.

Wenn Sie gemeinsame Vereinbarungen getroffen haben, äußeren Sie die Zuversicht, dass die getroffenen Vereinbarungen zu dem gewünschten Ergebnis führen.

\section{Phase 3: Die Nachbereitung}

Erstellen Sie zeitnah ein Protokoll, das durch Sie und den Mitarbeiter unterschrieben wird. Alle Vereinbarungen sind in schriftlicher Form, zweckmäßigerweise in entsprechenden Formblättern, festzuhalten und werden durch den Mitarbeiter und die Führungskraft unterschrieben. Beide erhalten eine Kopie des Aufzeichnungsbogens.

Grundsätzlich geht der Inhalt eines Mitarbeitergespräches nur Mitarbeiter und Vorgesetzte etwas an. In manchen Betrieben ist es aber üblich, das Ergebnis der Personalakte beizufügen oder dem Vorstand zur Kenntnis zu geben. Klären Sie dies vorab und klären Sie auch Ihre Mitarbeiterinnen und Mitarbeiter zu Beginn des Gespräches während der Informationsphase darüber auf, was wie dokumentiert wird. Gerade wenn Sie schwierige und unter Umständen die Persönlichkeit betreffende Sachverhalte (Perspektiven, Umgang mit bestimmten Situationen) erörtern, sollten Sie vielleicht zwei Protokolle erstellen. Ein offizielles Ergebnisprotokoll, das zur »Veröffentlichung « bestimmt ist sowie ein internes für Sie und Ihren Mitarbeiter, das als Gedächtnisstütze zur Vorbereitung des nächsten Gespräches dient. Sammeln Sie zudem die Protokolle nicht über Jahre hinweg. Sie erstellen kein Persönlichkeitsdossier sondern ein Arbeitsmittel. Spätestens wenn das darauffolgende Gespräch stattgefunden hat, sollte das vorherige Gesprächsprotokoll vernichtet werden. Alle dann noch offenen Fragen sollten Bestandteil des neuen Gespräches sein und sind dann aktualisiert im neuen Protokoll dokumentiert.

Beobachten Sie, wie sich die Situation weiter entwickelt. Wenn Sie erkennen, dass sich die avisierten Ziele nicht umsetzten lassen, führen Sie gegebenenfalls weitere Gespräche und treffen Sie eine neue Vereinbarung. Wenn die gewünschten Veränderungen eintreten: Machen Sie deutlich, dass sie dieses bemerkt haben. Verleihen Sie Ihrer Wertschätzung Ausdruck.

Und schließlich gilt: Nach dem Gespräch ist vor dem Gespräch. Führen Sie regelmäßig (mindestens jährlich) Zielvereinbarungs- und Entwicklungsgespräche mit Ihren Mitarbeiterinnen und Mitarbeitern. Nutzen Sie diese Instrumente der Personalführung regelmäßig, so werden Sie auf Dauer weniger Anlass für unangenehme anlassbezogene Kritikgespräche haben. 